\title{
Synthesis of New 5-arylazo-1-substitutedpyridone Dyes with Antibacterial Activity
}

\author{
Hatem E. Gaffer ${ }^{1}$, Mona E. Ibrahim ${ }^{2}$ Mahmoud M. Sultan² and Ehab Abdel-latif ${ }^{2}$ \\ ${ }^{1}$ Textile Research Division, National Research Centre, 12622 Dokki, Giza, Egypt \\ ${ }^{2}$ Department of Chemistry, Faculty of Science, Mansoura University, Mansoura 35516, Egypt.
}

\begin{abstract}
NEW SERIES of 5-arylazo-3-cyano-2-pyridone scaffolds 5, 6 and 7 have been synthesized and characterized by elemental analysis, IR and ${ }^{1} \mathrm{H}$ NMR spectroscopic tools. The antibacterial activities of the synthesized pyridone scaffolds have been investigated; they exhibited good activities compared to the standard drugs.
\end{abstract}

Keywords: 5-arylazo-2-pyridones, Cyanoacetamide, Phenacyl bromide, Chloroacetonitrile, Antibacterial activities.

\section{Introduction}

Pyridine ring represents the main skeleton of numerous bioactive molecules. Recently 2-pyridone and their derivatives take interest due to their anti-inflammatory, antiviral and antiproliferative activities [1-3]. Amrinone (I) [4] and Milrinone (II) [5] have been considered as cardiotonic drugs for the treatment of heart failure. Nowadays, 2-pyridone derivative (III) is investigated as a specific inhibitor of human immune deficiency virus-1 (HIV-1) [6]. Pyridone and its scaffolds have been reported as targeted molecules in many drug discovery programs of cancer and inflammatory disorders such as CDK4, FGFR and p38 inhibitors (IV-V) [7-10] respectively (Fig. 1).

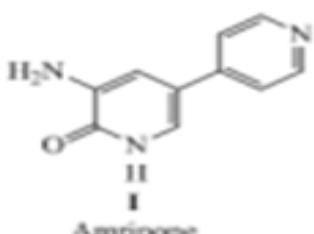<smiles>CC1=CC(c2ccncc2)C(C)N([N+]#[Co])C1=O</smiles><smiles>CCc1cc(NCc2nc3c(Cl)ccc(Cl)c3o2)c(=O)[nH]c1C</smiles><smiles>C=C(NC(=O)c1ccc(C)c(-c2cc3cnn(-c4c(F)cccc4F)c3n(C)c2=O)c1)C1CC1</smiles><smiles>C=C(NC1CC1)c1ccc(C)c(-c2cc3cnnc(-c4ccc(F)cc4F)c3n(C)c2=O)c1</smiles><smiles>CC(C)n1c(=O)ccc2cnc(Nc3ccc(N4CCN(C)CC4)cc3)nc21</smiles>

Fig. 1. 2-Pyridone scaffolds of medicinal interest.

\footnotetext{
*Corresponding author e-mail: hatem197@yahoo.com; Tel: 00201026896065

DOI: 10.21608/EJCHEM.2018.1508.1118

C2017 National Information and Documentation Center (NIDOC)
} 
Pyridone analogues were investigated for their in vitro antibacterial activity against Gram-positive bacteria (Staphylococcus aureus, Streptococcus pyogenes) and Gram-negative bacteria (Escherichia coli, Pseudomonas aeruginosa) [11]. Many hundreds of 2-pyridones have been prepared and evaluated in vitro and in vivo, and selected members are advancing toward human clinical trials [12]. A series of novel pyridone-based disperse disazo dyes was synthesized by diazotizing and coupling reactions for dyeing polyester fabric [13]. The solvatochromic properties and antimicrobial activities of these pyridonebased disperse disazo dyes in various solvents were evaluated [14]. Therefore, the construction of 2-pyridones has been acquired much pharmaceutical importance in recent years [15-20].

\section{Experimental}

Melting points (uncorrected) were measured on Gallenkamp electric melting point apparatus. Infrared spectra were determined on Mattson 5000
FT-IR spectrometer ( $\mathrm{KBr}$ discs). ${ }^{1} \mathrm{H}$ NMR spectra were recorded on a Bruker WP spectrometer (USA) (300 MHz) using TMS as an internal standard. Elemental analyses $(\mathrm{C}, \mathrm{H}$ and $\mathrm{N})$ were determined on Perkin-Elmer 2400 analyzer.

Synthesis of 5-arylazo-3-cyano-1-(ethoxycarbonyl) methyl-4,6-dimethyl-2-pyridone dyes 5, 6 and 7

To a suspension of 5-arylazopyridones 3 $(0.005 \mathrm{~mol})$ in DMF $(20 \mathrm{ml}), 0.005 \mathrm{~mol}$ of the appropriate alpha-halogenated reagent (namely; ethyl bromoacetate, phenacyl bromide and/ or chloroacetonitrile) was added. The reaction suspension was heated at $90^{\circ} \mathrm{C}$ with stirring for 4 hours and then poured into ice-water drop by drop. The crude product, which picked up by filtration, was purified by recrystallization in ethyl alcohol to furnish the 2-pyridone dyes 5, 6 and 7, respectively.

3-Cyano-1-(ethoxycarbonyl)methyl-4,6dimethyl-5-phenylazo-2-pyridone (5a) was isolated as orange crystals.

TABLE 1. Physicochemical data for the synthesized 5-arylazo-2-pyridone scaffolds 5-7.

\begin{tabular}{|c|c|c|c|c|c|c|c|}
\hline \multirow{2}{*}{ Cpd. } & \multirow{2}{*}{ Molecular formula } & \multirow{2}{*}{ MW } & \multirow{2}{*}{$\mathbf{M P},{ }^{\circ} \mathrm{C}$} & \multirow{2}{*}{$\begin{array}{c}\text { Yield, } \\
\%\end{array}$} & \multicolumn{3}{|c|}{ Analysis \%, Calcd. (Found) } \\
\hline & & & & & C & $\mathbf{H}$ & $\mathbf{N}$ \\
\hline \multirow[b]{2}{*}{$5 a$} & \multirow[b]{2}{*}{$\mathrm{C}_{18} \mathrm{H}_{18} \mathrm{~N}_{4} \mathrm{O}_{3}$} & \multirow[b]{2}{*}{338} & \multirow[b]{2}{*}{$114-115$} & \multirow[b]{2}{*}{81} & 63.89 & 5.36 & 16.56 \\
\hline & & & & & (63.74) & (5.42) & (16.67) \\
\hline \multirow{2}{*}{$5 b$} & \multirow[b]{2}{*}{$\mathrm{C}_{19} \mathrm{H}_{20} \mathrm{~N}_{4} \mathrm{O}_{3}$} & \multirow{2}{*}{352} & \multirow{2}{*}{$117-118$} & \multirow{2}{*}{63} & 64.76 & 5.72 & 15.90 \\
\hline & & & & & 64.95 & 5.76 & 15.82 \\
\hline \multirow{2}{*}{$5 c$} & \multirow{2}{*}{$\mathrm{C}_{18} \mathrm{H}_{17} \mathrm{ClN}_{4} \mathrm{O}_{3}$} & \multirow{2}{*}{372} & \multirow{2}{*}{$122-123$} & \multirow{2}{*}{63} & 57.99 & 4.60 & 15.03 \\
\hline & & & & & (58.17) & $(4.55)$ & (15.15) \\
\hline \multirow[b]{2}{*}{$6 a$} & \multirow[b]{2}{*}{$\mathrm{C}_{22} \mathrm{H}_{18} \mathrm{~N}_{4} \mathrm{O}_{2}$} & \multirow[b]{2}{*}{370} & \multirow[b]{2}{*}{$138-140$} & \multirow[b]{2}{*}{55} & 71.34 & 4.90 & 15.13 \\
\hline & & & & & (71.54) & (4.96) & $(15.21)$ \\
\hline \multirow{2}{*}{$6 \mathbf{b}$} & \multirow[b]{2}{*}{$\mathrm{C}_{23} \mathrm{H}_{20} \mathrm{~N}_{4} \mathrm{O}_{2}$} & \multirow{2}{*}{384} & \multirow{2}{*}{$158-160$} & \multirow{2}{*}{43} & 71.86 & 5.24 & 14.57 \\
\hline & & & & & (71.72) & (5.19) & (14.64) \\
\hline \multirow{2}{*}{$6 c$} & \multirow{2}{*}{$\mathrm{C}_{22} \mathrm{H}_{17} \mathrm{ClN}_{4} \mathrm{O}_{2}$} & \multirow{2}{*}{404} & \multirow{2}{*}{ 153-155 } & & 65.27 & 4.23 & 13.69 \\
\hline & & & & 70 & $(65.47)$ & (4.31) & (13.84) \\
\hline 7 & CUIS & & & & 65.97 & 4.50 & 24.04 \\
\hline $7 \mathbf{a}$ & $\mathrm{C}_{16} \mathrm{H}_{13} \mathrm{~N}_{5} \mathrm{O}$ & 291 & $224-225$ & 45 & $(66.15)$ & (4.43) & (23.95) \\
\hline & $C$ & & & & 66.87 & 4.95 & 22.94 \\
\hline $7 \mathbf{D}$ & $\mathrm{C}_{17} \mathrm{H}_{15} \mathrm{~N}_{5} \mathrm{U}$ & 305 & $223-225$ & 40 & $(66.77)$ & (4.89) & (22.88) \\
\hline & & & & & 58.99 & 3.71 & 21.50 \\
\hline $7 \mathrm{c}$ & $\mathrm{C}_{16} \mathrm{H}_{12} \mathrm{ClN}_{5} \mathrm{O}$ & 325 & $220-222$ & 53 & $(58.78)$ & $(3.76)$ & $(21.60)$ \\
\hline
\end{tabular}


3-Cyano-1-(ethoxycarbonyl)methyl-4,6dimethyl-5-(4-tolylazo)-2-pyridone (5b) was isolated as orange crystals.

5 - (3-Chlorophenylazo) - 3-cyano- 1 (ethoxycarbonyl)methyl-4,6-dimethyl-2pyridone $(5 \mathrm{c})$ was isolated as orange crystals,

3-Cyano-4,6-dimethyl-1-(2-oxo-2-phenylethyl)5-phenylazo-2-pyridone (6a) was isolated as brown crystals.

3-Cyano-4,6-dimethyl-1-(2-oxo-2-phenylethyl)5-(4-tolylazo)-2-pyridone (6b) was isolated as reddish brown crystals.
5-(3-Chlorophenylazo)-3-cyano-4,6-dimethyl1-(2-oxo-2-phenylethyl)- 2-pyridone (6c) was isolated as light brown crystals.

3-Cyano-1-(cyanomethyl)-4,6-dimethyl-5phenylazo-2-pyridone (7a) was isolated as lightgreen crystals.

3-Cyano-1-(cyanomethyl)-4,6-dimethyl-5-(4tolylazo)-2-pyridone (7b) was isolated as orange crystals.

5 - (3 - Chlorophenylazo) - 3 - cyano- 1 (cyanomethyl)-4,6-dimethyl-2-pyridone (7c) was isolated as green crystals.

TABLE 2. Spectral data for the synthesized 5-arylazo-2-pyridone scaffolds 5-7.

\begin{tabular}{|c|c|c|}
\hline Cpd. & IR $(\mathrm{KBr}), \mathbf{c m}^{-1}$ & ${ }^{1} \mathrm{H}$ NMR (DMSO-d $): \delta(\mathrm{ppm})$ \\
\hline $5 \mathbf{a}$ & $\begin{array}{l}2224(\mathrm{C} \equiv \mathrm{N}), \\
1752(\mathrm{C}=\mathrm{O}), \\
1652(\mathrm{C}=\mathrm{O})\end{array}$ & $\begin{array}{l}1.20\left(\mathrm{t}, 3 \mathrm{H}, \mathrm{CH}_{3}\right), 2.52\left(\mathrm{~s}, 3 \mathrm{H}, \mathrm{CH}_{3}\right), 2.60\left(\mathrm{~s}, 3 \mathrm{H}, \mathrm{CH}_{3}\right), 4.21\left(\mathrm{q}, 2 \mathrm{H}, \mathrm{CH}_{2}\right), 5.11 \\
\left(\mathrm{~s}, 2 \mathrm{H}, \mathrm{CH}_{2}\right), 7.16-7.45(\mathrm{~m}, 5 \mathrm{H}, \mathrm{Ar}-\mathrm{H}) .\end{array}$ \\
\hline $5 \mathbf{b}$ & $\begin{array}{l}2226(\mathrm{C} \equiv \mathrm{N}), \\
1754(\mathrm{C}=\mathrm{O}), \\
1644(\mathrm{C}=\mathrm{O})\end{array}$ & $\begin{array}{l}1.21\left(\mathrm{t}, 3 \mathrm{H}, \mathrm{CH}_{3}\right), 2.42\left(\mathrm{~s}, 3 \mathrm{H}, \mathrm{CH}_{3}\right), 2.50\left(\mathrm{~s}, 3 \mathrm{H}, \mathrm{CH}_{3}\right), 2.57\left(\mathrm{~s}, 3 \mathrm{H}, \mathrm{CH}_{3}\right), \\
4.19\left(\mathrm{q}, 2 \mathrm{H}, \mathrm{CH}_{2}\right), 5.09\left(\mathrm{~s}, 2 \mathrm{H}, \mathrm{CH}_{2}\right), 7.42(\mathrm{~d}, 2 \mathrm{H}, \mathrm{Ar}-\mathrm{H}), 7.78(\mathrm{~d}, 2 \mathrm{H}, \mathrm{Ar}-\mathrm{H}) .\end{array}$ \\
\hline $5 c$ & $\begin{array}{l}2223(\mathrm{C} \equiv \mathrm{N}) \\
1752(\mathrm{C}=\mathrm{O}) \\
1657(\mathrm{C}=\mathrm{O})\end{array}$ & $\begin{array}{l}1.21\left(\mathrm{t}, 3 \mathrm{H}, \mathrm{CH}_{3}\right), 2.55\left(\mathrm{~s}, 3 \mathrm{H}, \mathrm{CH}_{3}\right), 2.62\left(\mathrm{~s}, 3 \mathrm{H}, \mathrm{CH}_{3}\right), 4.20\left(\mathrm{q}, 2 \mathrm{H}, \mathrm{CH}_{2}\right), 5.11 \\
\left(\mathrm{~s}, 2 \mathrm{H}, \mathrm{CH}_{2}\right), 7.61-7.88(\mathrm{~m}, 4 \mathrm{H}, \mathrm{Ar}-\mathrm{H}) .\end{array}$ \\
\hline $6 a$ & $\begin{array}{l}2224(\mathrm{C} \equiv \mathrm{N}) \\
1698(2 \mathrm{C}=\mathrm{O})\end{array}$ & $\begin{array}{l}2.45\left(\mathrm{~s}, 3 \mathrm{H}, \mathrm{CH}_{3}\right), 2.55\left(\mathrm{~s}, 3 \mathrm{H}, \mathrm{CH}_{3}\right), 5.90\left(\mathrm{~s}, 2 \mathrm{H}, \mathrm{CH}_{2}\right), 7.15-7.68(\mathrm{~m}, 10 \mathrm{H} \text {, } \\
\mathrm{Ar}-\mathrm{H}) .\end{array}$ \\
\hline $6 b$ & $\begin{array}{l}2223(\mathrm{C} \equiv \mathrm{N}), \\
1699(\mathrm{C}=\mathrm{O}), \\
1650(\mathrm{C}=\mathrm{O})\end{array}$ & $\begin{array}{l}2.38\left(\mathrm{~s}, 3 \mathrm{H}, \mathrm{CH}_{3}\right), 2.40\left(\mathrm{~s}, 3 \mathrm{H}, \mathrm{CH}_{3}\right), 2.58\left(\mathrm{~s}, 3 \mathrm{H}, \mathrm{CH}_{3}\right), 5.95\left(\mathrm{~s}, 2 \mathrm{H}, \mathrm{CH}_{2}\right), \\
7.33-8.03(\mathrm{~m}, 9 \mathrm{H}, \mathrm{Ar}-\mathrm{H}) .\end{array}$ \\
\hline $6 c$ & $\begin{array}{l}2225(\mathrm{C} \equiv \mathrm{N}), \\
1699(\mathrm{C}=\mathrm{O}), \\
1662(\mathrm{C}=\mathrm{O})\end{array}$ & $\begin{array}{l}2.44\left(\mathrm{~s}, 3 \mathrm{H}, \mathrm{CH}_{3}\right), 2.64\left(\mathrm{~s}, 3 \mathrm{H}, \mathrm{CH}_{3}\right), 5.98\left(\mathrm{~s}, 2 \mathrm{H}, \mathrm{CH}_{2}\right), 7.58-8.04(\mathrm{~m}, 9 \mathrm{H} \text {, } \\
\text { Ar-H). }\end{array}$ \\
\hline $7 \mathbf{a}$ & $\begin{array}{l}2222(\mathrm{C} \equiv \mathrm{N}) \\
1656(\mathrm{C}=\mathrm{O})\end{array}$ & $\begin{array}{l}2.59\left(\mathrm{~s}, 3 \mathrm{H}, \mathrm{CH}_{3}\right), 2.64\left(\mathrm{~s}, 3 \mathrm{H}, \mathrm{CH}_{3}\right), 5.42\left(\mathrm{~s}, 2 \mathrm{H}, \mathrm{CH}_{2}\right), 7.51-7.78(\mathrm{~m}, 5 \mathrm{H} \text {, } \\
\text { Ar-H). }\end{array}$ \\
\hline $7 b$ & $\begin{array}{l}2223(\mathrm{C} \equiv \mathrm{N}) \\
1657(\mathrm{C}=\mathrm{O})\end{array}$ & $\begin{array}{l}2.38\left(\mathrm{~s}, 3 \mathrm{H}, \mathrm{CH}_{3}\right), 2.56\left(\mathrm{~s}, 3 \mathrm{H}, \mathrm{CH}_{3}\right), 2.61\left(\mathrm{~s}, 3 \mathrm{H}, \mathrm{CH}_{3}\right), 5.34\left(\mathrm{~s}, 2 \mathrm{H}, \mathrm{CH}_{2}\right), 7.36 \\
(\mathrm{~d}, 2 \mathrm{H}, \mathrm{Ar}-\mathrm{H}), 7.70(\mathrm{~d}, 2 \mathrm{H}, \mathrm{Ar}-\mathrm{H}) .\end{array}$ \\
\hline $7 \mathrm{c}$ & $\begin{array}{l}2222(\mathrm{C} \equiv \mathrm{N}) \\
1654(\mathrm{C}=\mathrm{O})\end{array}$ & $\begin{array}{l}{ }^{1} \mathrm{H} \text { NMR: } 2.58\left(\mathrm{~s}, 3 \mathrm{H}, \mathrm{CH}_{3}\right), 2.64\left(\mathrm{~s}, 3 \mathrm{H}, \mathrm{CH}_{3}\right), 5.38\left(\mathrm{~s}, 2 \mathrm{H}, \mathrm{CH}_{2}\right), 7.26-7.80 \\
(\mathrm{~m}, 4 \mathrm{H}, \mathrm{Ar}-\mathrm{H}) .\end{array}$ \\
\hline
\end{tabular}




\section{Antimicrobial activity}

organic compounds were evaluated against (Escherichia coli) as a gram negative and (Staphylococcus aureus) as a gram positive bacteria as well as Candida albicans fungus (yeast) strain. Antimicrobial activity were examined by the agar well diffusion method using $100 \mu \mathrm{L}$ of suspension containing $1 \times 10^{8} \mathrm{CFU} / \mathrm{mL}$ of pathological tested bacteria [12], antimicrobial activity was evaluated by measuring the zone of inhibition against the test organisms and compared with that of the standard.

\section{Results and Discussion}

The current synthetic strategy of 5-arylazopyridone dyes 5, 6 and 7 starts through heterocyclization of three arylazo derivatives of acetylacetone 1 with cyanoacetamide 2 by heating in ethanol containing potassium hydroxide to furnish the corresponding potassium salt of 5-arylazo-pyridones 3. Alkylation of these pyridone potassium salts with ethyl bromoacetate in DMF at $90^{\circ} \mathrm{C}$ proceeded at the more nucleophilic nitrogen atom of the pyridine ring to afford the N-alkylated product, 5-arylazo1-(ethoxycarbonyl)methyl-pyridones 5. The reaction failed to give the O-alkylated product 4 which may be obtained by alkylation at the less nucleophilic oxygen atom. The chemical structure of 5a-c was secured base on the correct elemental analysis and spectral data. The IR spectrum of 5-phenylazo-2-pyridone 5a revealed absorption bands at $2224 \mathrm{~cm}^{-1}$ for the nitrile function $(\mathrm{C} \equiv \mathrm{N})$ and $1752 \& 1652 \mathrm{~cm}^{-1}$ to indicate the carbonyl functions $(2 \mathrm{C}=\mathrm{O})$. The ${ }^{1} \mathrm{H}$ NMR spectrum exhibited singlet signal at $5.11 \mathrm{ppm}$ to secure the protons of methylene group $\left(\mathrm{CH}_{2}\right)$.

Similar treatment of 5-arylazo-pyridones 3 with phenacyl bromide and/or chloroacetonitrile in $\mathrm{DMF}$ at $90^{\circ} \mathrm{C}$ furnished the corresponding 5-arylazo-1-(2-oxo-2-phenylethyl)-pyridones 6 and 5-arylazo-1-(cyanomethyl)-pyridones 7, respectively. The chemical structures of these pyridone dyes was secured by their correct elemental and spectral analyses. The IR spectra of 5-arylazo-1-(2-oxo-2-phenylethyl)pyridones 6a-c revealed the absorption band of carbonyl function $\left(\mathrm{NCH}_{2} \mathrm{COPh}\right)$ at 1698 or $1699 \mathrm{~cm}^{-1}$ while that of nitrile function $(\mathrm{C} \equiv \mathrm{N})$ in the range from 2223 to $2225 \mathrm{~cm}^{-1}$. The ${ }^{1} \mathrm{H}$ NMR spectra of these pyridones $6 \mathrm{a}-\mathrm{c}$ exhibited the protons of methylene group $\left(\mathrm{N}-\mathrm{CH}_{2} \mathrm{COPh}\right)$ as singlet near $5.95 \mathrm{ppm}$. The ${ }^{1} \mathrm{H}$ NMR spectra of 5-arylazo1-(cyanomethyl)-pyridones $7 \mathrm{a}-\mathrm{c}$ exhibited the protons of methylene group $\left(\mathrm{N}-\mathrm{CH}_{2} \mathrm{CN}\right)$ singlet in the region 5.34-5.42 ppm.

TABLE 3. inhibition zone Diameters against a variety of bacteria and fungi (growth after 2 days). ${ }^{a}$

\begin{tabular}{cccc}
\hline & \multicolumn{3}{c}{ Diameter inhibition zone in mm (\% activity index $)$} \\
\hline Compd. No. & E. coli & S. aureus & C. albicans \\
$5 \mathrm{a}$ & $20(83)$ & $15(68)$ & $23(82)$ \\
$5 \mathrm{~b}$ & $16(67)$ & $\mathrm{b}$ & $11(39)$ \\
$5 \mathrm{c}$ & $\mathrm{b}$ & $\mathrm{b}$ & $10(43)$ \\
$6 \mathrm{a}$ & $\mathrm{b}$ & $17(77)$ & $20(71)$ \\
$6 \mathrm{~b}$ & $\mathrm{~b}$ & $\mathrm{~b}$ & $11(42)$ \\
$6 \mathrm{c}$ & $9(38)$ & $11(50)$ & $16(59)$ \\
$7 \mathrm{a}$ & $24(100)$ & $11(50)$ & $16(57)$ \\
$7 \mathrm{~b}$ & $15(57)$ & $22(100)$ & $9(33)$ \\
$7 \mathrm{c}$ & $18(69)$ & $18(65)$ & $26(100)$ \\
\hline
\end{tabular}

\footnotetext{
${ }^{a}$ Diameters (mm) of zones of inhibition (agar diffusion assay) are provided. Ampicillin and colitrimazole were used as the positive control.
}

${ }^{\mathrm{b}}$ Values below $6 \mathrm{~mm}(25 \%)$ are of limited value as they refer either to inactive or non-diffusing compounds. 


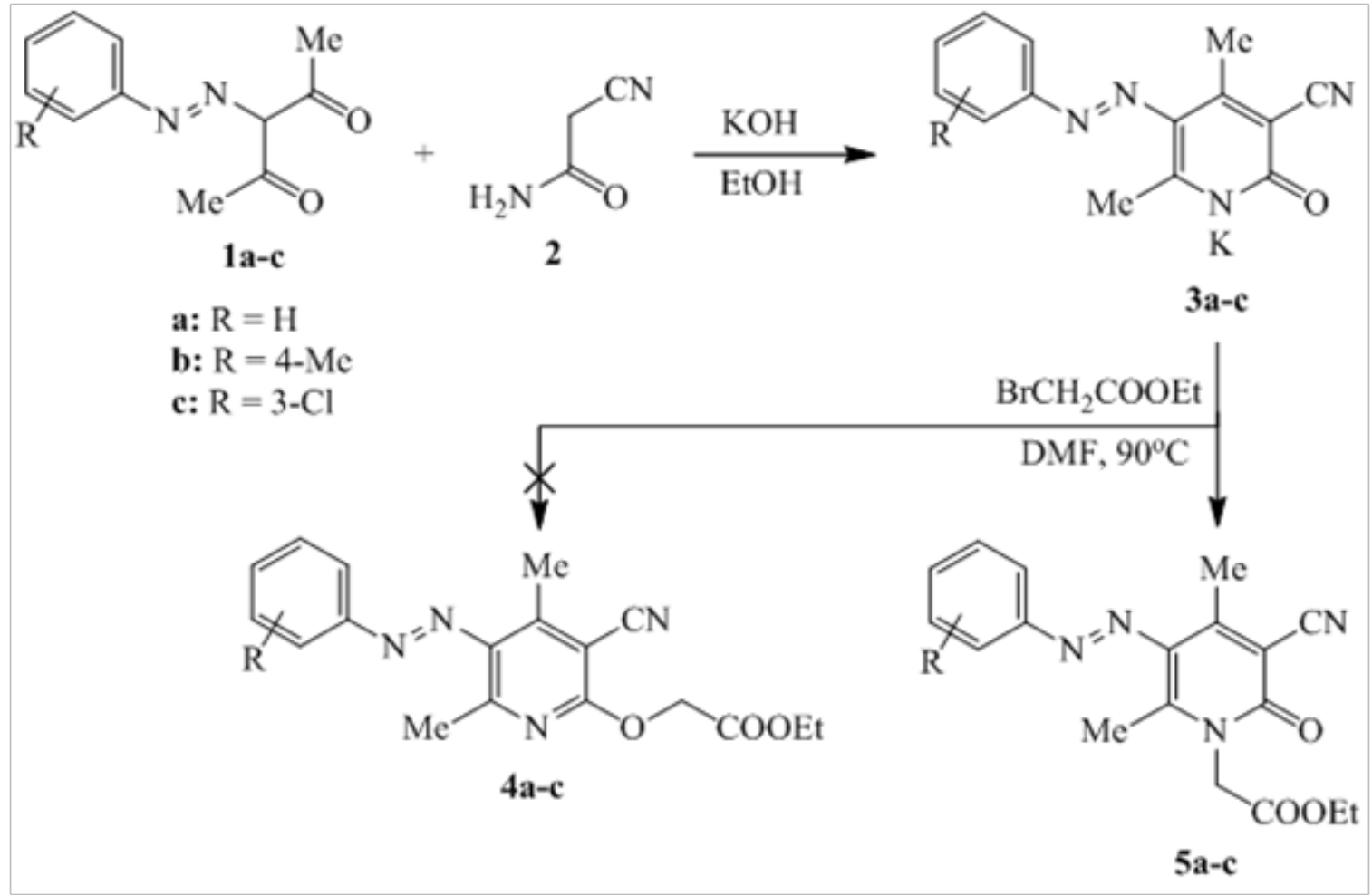

Scheme 1. Synthesis of 5-arylazo-1-(ethoxycarbonyl)methyl-2-pyridones 5a-c.

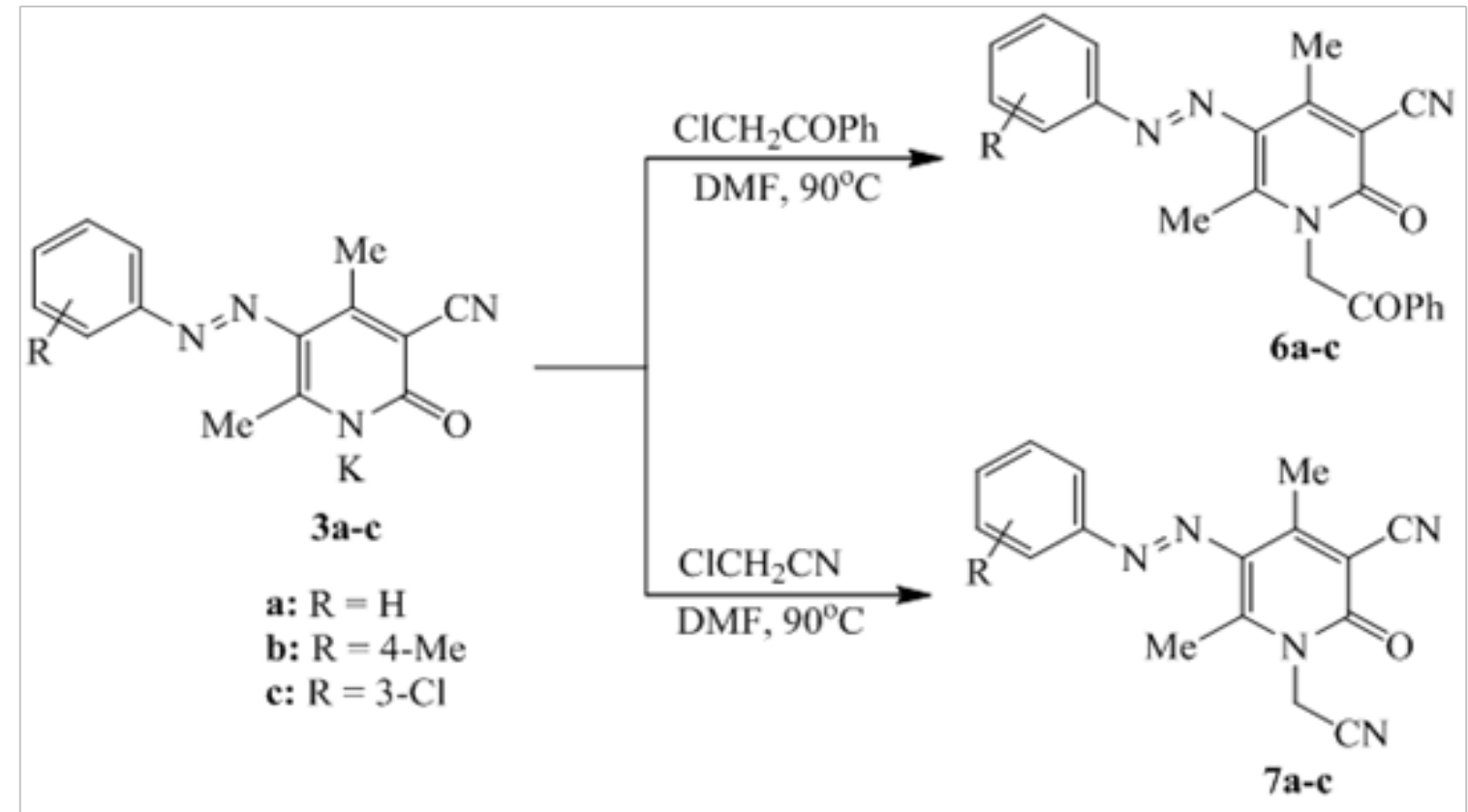

Scheme 2. Synthesis of 5-arylazo-1-(2-oxo-2-phenylethyl)-2-pyridones 6a-c and 5-arylazo-1-(cyanomethyl)-2pyridones 7 a-c. 


\section{Conclusion}

Newly series of 5-arylazo-3-cyano-4,6dimethyl-2-pyridone scaffolds 5, 6 and 7 have been synthesized and elucidated by elemental analysis, IR and 1H NMR spectroscopic tools. The satisfactory synthetic dyes performance and good antibacterial properties should lead to design of novel antibacterial disperse dyes with improved application properties.

\section{Funding}

This research received no specific grant from any funding agency in the public, commercial, or not-for-profit sectors.

\section{Acknowledgment}

Authors would like to thank Dr. Khaled Elattar, Division of Biochemistry, Faculty of Science, Mansoura University (NRC), Cairo, Egypt for their excellent technical assistance during the antimicrobial evaluation of the synthetic dyes.

\section{References}

1. Choi W.B., Houpis I.N., Churchill H.R., Molina A., Lynch J.E., Volante R.P., Reider P.J. and King A.O., A practical synthesis of the 5-chloromethylfuro[2,3-b]pyridine pharmacophore. Tetrahedron letters, 36, 4571 (1995).

2. Bhupathy M., Conlon D.A., Wells K.M., Nelson J.R., Reider P.J., Rossen K., Sager J.W., Volante R.P., Dorsey B.D., Hoffman J.M. and Joseph,S.A., A practical synthesis of 5-(chloromethyl) furo [2,3- $b]$ pyridine, a key intermediate for the HIV protease inhibitor, L-754,394. Journal of heterocyclic chemistry, 32, 1283 (1995).

3. Kappe C.O. and Kappe T., Synthesis of substituted 3-pyridinecarbonitriles with potential biological activity. Monatshefte für Chemiel Chemical Monthly, 12, 1095 (1989).

4. Pastelin G., Mendez R., Kabela E. and Farah A., The search for a digitalis substitute II milrinone (Win 47203) its action on the heart-lung preparation of the dog. Life sciences, 33, 1787 (1983).

5. Altomare C., Cellamare S., Summo L., Fossa P., Mosti L. and Carotti A., Ionization behaviour and tautomerism-dependent lipophilicity of pyridine-2 (1H)-one cardiotonic agents. Bioorganic \& medicinal chemistry, 8, 909 (2000).

6. Parreira R.L., Abrahão O. and Galembeck S.E., Conformational preferences of nonnucleoside HIV-1 reverse transcriptase inhibitors. Tetrahedron, 57, 3243 (2001).
7. Pettus L.H., Wurz R.P., Xu S., Herberich B., Henkle B., Liu Q., McBride H.J., Mu S., Plant M.H., Saris C.J. and Sherman L., Discovery and evaluation of 7-alkyl-1, 5-bis-arylpyrazolopyridinones as highly potent, selective, and orally efficacious inhibitors of p $38 \alpha$ mitogenactivated protein kinase $\perp \perp$ atomic coordinates and structure factors for crystal structure of compound $3 \mathrm{~d}$ with $\mathrm{p} 38 \alpha$ can be accessed using PDB code 3LHJ. Journal Of Medicinal Chemistry, 53, 2973 (2010).

8. Pettus Liping H., et al. Discovery and evaluation of 7-alkyl-1, 5-bis-aryl-pyrazolopyridinones as highly potent, selective, and orally efficacious inhibitors of $\mathrm{p} 38 \alpha$ mitogen-activated protein kinase $п$ atomic coordinates and structure factors for crystal structure of compound $3 \mathrm{~d}$ with $\mathrm{p} 38 \alpha$ can be accessed using PDB code 3LHJ. Journal Of Medicinal Chemistry, 53, 2973 (2010).

9. Milburn R.R., Thiel O.R., Achmatowicz M., Wang X., Zigterman J., Bernard C., Colyer J.T., DiVirgilio E., Crockett R., Correll T.L. and Nagapudi K., Development of a practical synthesis of a pyrazolopyridinone-based $\mathrm{p} 38$ map kinase inhibitor. Organic Process Research \& Development, 15, 31 (2010).

10. Barvian M., Boschelli D.H., Cossrow J., Dobrusin E., Fattaey A., Fritsch A., Fry D., Harvey P., Keller P., Garrett M. and La F., Pyrido pyrimidin-7-one inhibitors of cyclin-dependent kinases. Journal Of Medicinal Chemistry, 43, 4606 (2000).

11. Desai N.C., Rajpara K.M. and Joshi V.V., Synthesis of pyrazole encompassing 2-pyridone derivatives as antibacterial agents. Bioorganic \& Medicinal Chemistry Letters, 23, 271(2013).

12. Li Q., Mitscher L.A. and Shen L.L., The 2-pyridone antibacterial agents: bacterial topoisomerase inhibitors. Medicinal Research Reviews, 20, 231(2000).

13. Metwally M.A., Etman H.A., Gaffer H.E. and Khalil A.M., The use of 3-amino-4, 6-dimethylpyrazolo pyridine in the synthesis of novel heterocycles of pharmaceutical interest. Chemistry Of Heterocyclic Compounds. 44, 715 (2008).

14. Karcı F., Demirçalı A. and Yamaç M., Synthesis, solvatochromic properties and antimicrobial activities of some novel pyridone-based disperse disazo dyes. Journal of Molecular Liquids. 187, 302 (2013).

15. Kaneko M., Yamamoto H., Sakai H., KamadaY., 
Tanaka T., Fujiwara S., Yamamoto S., Takahagi H., Igawa H., Kasai S., Noda M., Inui M. and Nishimoto T., A pyridone derivative activates SERCA2a by attenuating the inhibitory effect of phospholamban. European Journal of Pharmacology. 2017, http://dx.doi.org/10.1016/j. ejphar.2017.07.035

16. Abouzid K.A.M., Al-Ansary G.H. and ElNaggar A.M., Eco-friendly synthesis of novel cyanopyridine derivatives and their anticancer and PIM-1 kinase inhibitory activities. European Journal of Medicinal Chemistry, 134, 357 (2017).

17. Sheibani H., Saidi K., Abbasnejad M., Derakhshani A. and Mohammadzadeh I., A convenient one-pot synthesis and anxietic activity of 3-cyano-2(1H)-iminopyridines and halogen derivatives of benzo $[h]$ chromenes. Arabian Journal of Chemistry, 9, s901 (2016).

18. Buduma K., Chinde S., Arigari N.K., Grover P., Srinivas K.V.N.S. and Kumar J. K., Iodine catalyzed simple and efficient synthesis of antiproliferative 2-pyridones. Bioorganic \& Medicinal Chemistry Letters, 26, 2159-2163 (2016).

19. Helal M.H., El-Awdan S.A., Salem M.A., Abdelaziz T.A., Moahamed Y.A., El-Sherif A.A. and Mohamed G.A.M., Synthesis, biological evaluation and molecular modeling of novel series of pyridine derivatives as anticancer, anti-inflammatory and analgesic Agents. Spectrochimica Acta Part A: Molecular and Biomolecular Spectroscopy, 135, 764 (2015).

20. Ohishi K., Toume K., Arai M.A., Sadhu S.K., Ahmed F., Mizoguchi T., Itoh M. and Ishibashi M., Ricinine: A pyridone alkaloid from Ricinus communis that activates the Wnt signaling pathway through casein kinase $1 \alpha$., Bioorg. Med. Chem., 22, 4597-4601 (2014).

(Received 22/8/2017; accepted 10/1/2018)

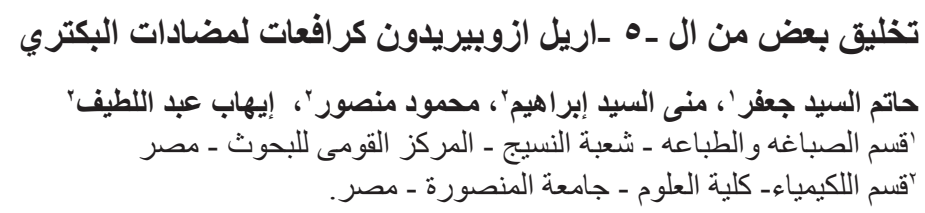

